\title{
WHAT TO DO WHEN YOU DO NOT SEE THE DATA
}

\author{
Bruno de Sousa $^{1}$, Rosário Gomes ${ }^{2}$, Luís Barata $^{2}$ and Afonso Domingos ${ }^{3}$ \\ ${ }^{1}$ Faculty of Psychology and Education Sciences, CINEICC, University of Coimbra, Portugal \\ ${ }^{2}$ Media Production Center - Audio and Braille, University of Coimbra, Portugal \\ ${ }^{3}$ Faculty of Psychology and Education Sciences, University of Coimbra, Portugal \\ bruno.desousa@fpce.uc.pt
}

\begin{abstract}
What recourse exists when the teaching of Statistics by visual means is not part of the equation? This case study of a team from the University of Coimbra - one teacher, two members of the Media Production Center and a visually impaired student - took up the challenge of teaching a full semester in Statistics in such a way that all students would experience and comprehend fundamental concepts ranging from summarizing data numerically and graphically to making decisions in Statistics through understanding centrality, dispersion and hypothesis testing. A very pragmatic non-visual orientation required class planning to emphasize on contents received aurally or read in Braille, with activities and challenges designed to engage all students in a variety of formats. Materials used in class will be presented in which Statistical Thinking is explored using visual, aural and tactile senses. Future research opportunities will be discussed.
\end{abstract}

\section{INTRODUCTION}

We all know how challenging it can be to teach Statistics to service courses. Many of these students have little or no knowledge in mathematics, and a significant number of them suffer from math phobia, or at the very least some display clear anxiety, tension and apprehension when dealing with numbers. It may be said that Statistics is "a nice version of Mathematics," but when learning software is required, either a very user-friendly option such as SPSS or a more programing oriented one such as $\mathrm{R}$, the learner stress rises considerably. Students complain about how difficult the subject matter is, and teachers become quite frustrated trying to reach out to students and make them understand how certain basic knowledge of Statistics can enable them to reflect, criticize, interpret and participate in society as well-informed citizens in both their own country and the wider world.

The core team of the present work is as follows: two normal visual individuals (the teacher and one member from the Media Production Center), one blind individual from the Media Production Center, and one visually impaired (95\%) student. The combination of a teacher with no experience in teaching Statistics to special needs individuals and a support staff with no background in Statistics together with a student eager to learn - created the perfect environment to envision Statistical Thinking in a way in which visual, aural and tactile senses were employed together. This took place within the context of a class with 104 students, who from the beginning demonstrated strong support for the visually impaired student. The Media Production Center undertook the production of the Braille texts and all the embossed printings, where the blind staff member was responsible for the Braille texts and the normal visual staff, together with the teacher, were responsible for adapting the graphical figures into the embossed printings and revising all the materials.

Before presenting examples of audio excerpts, embossed printings, $R$ programming scripts and Multiplano, a didactic tactile device used in this process, it is important to mention why a simple translation of the lecture contents and notes into Braille will not be sufficient to ensure a satisfying inclusive environment in class. The following text represents the 10 lines of the Abstract of this paper in Braille, in a font-size ready to be printed and given to a visually impaired person, with the gray shaded symbols indicating the beginning of each sentence:

\section{What recourse exists when the teaching of statistics by visual means is not part of the equation? This case study of a team from the University of Coimbra- one teacher, two members of the Media Production Center}


and a visually impaired student - took up the challenge of teaching a full semester in Statistics in such a way that all students would experience and comprehend fundamental concepts ranging from summarizing data numerically and graphically to making decisions in Statistics through understanding centrality, dispersion and hypothesis testing. A very pragmatic non-visual orientation required class planning to emphasize on contents received aurally or read in Braille, with activities and challenges designed to engage all students in a variety of formats. Materials used in class will be presented in which Statistical Thinking is explored using visual, aural and tactile senses. Future research opportunities will be discussed.

With the understanding of how simply producing texts written in Braille would be cumbersome and impractical (note how a mere 10 lines of text extends beyond a single A4 page), it became immediately clear that alternatives ways of teaching statistical concepts would become vital. Would the implementation of our motto - "If you do not see the data, try feeling it." - actually help not only the visually impaired students but also the remaining students to grasp statistical concepts? This is the research question that grew out of this unforgettable experience.

In the following sections, a series of different activities performed in class will be presented, together with reflections and a discussion of the shortcomings and future research opportunities in this area.

USING VISUAL, AURAL AND TACTILE SENSES

The four activities that will be described below were performed in class and directed at all students, with some adaptations made to allow for the participation of the special need student.

\section{Undesirable variability}

One of the fundamental objectives of Statistics is not to eliminate variability but to seek knowledge from studying the right kind of variability. How do you convey this idea to students, i.e. how to focus on the right kind of variability and avoid the spurious and systematic one sometimes generated in research?

In Figure 1 we can see two parts of a very simple experiment where we aim to explain how spurious variability can emerge from a wrongly planned experiment, and how sometimes simple solutions may avoid this undesirable variability. 


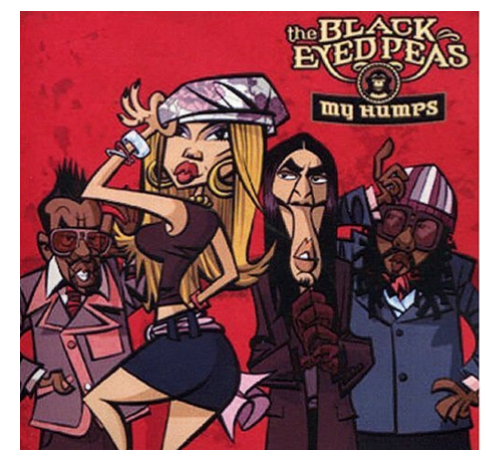

What you gon' do with all that junk?

All that junk inside your trunk?

I'm a get, get, get, get, you drunk,

Get you love drunk off my humps.

My humps, my humps, my humps, my humps, my humps,

My humps, my humps, my humps, my lovely little lumps.

Check it out

Figure 1. Part 1 (left) and Part 2 (right) of the Black Eyed Peas experiment

In the first part, by clicking on the Black Eyed Peas image (left of Figure 1), students will hear and see 1 minute and 46 seconds of the video music "My Humps". After viewing/listening, they are asked the question "How many times did you hear the word humps?" After the inevitable student complaints of unfairness for not having been given the question prior to the video, they start guessing at the number of the word humps, which range from as few as 10 to the unrealistic number of 110 , indeed quite far from the actual number of 21 repetitions. Students almost immediately came to the conclusion that if the question was made prior to the video being shown, the answers would be quite different.

In the second part of the experiment (right of Figure 1), students are given a written excerpt of "My Humps" (Latin alphabet or Braille) and asked, prior to the text being shown for 30 seconds, to count the number of letters " $u$ " in the text. The responses are very clear now, ranging from 18 to 21 , much closer to the true number of 20 Us in the text.

At the end, students clearly see the very different types of variability generated from the two parts of this activity. This very simple activity allows us to further explore and discuss different scenarios from real-life studies where undesirable variability can arise when research plans have not been carefully made. For example, applying questionnaires that were not tested prior to being given to participants may create undesirable variability when questions are not clearly understood by participants.

\section{Representative sample}

The second activity was one that I learned with a master teacher, Martha Aliaga (Aliaga \& Gunderson, 2016), centering on understanding how representative a sample is from a population. Picking up two neck scarves used in class, one of a single color and the other with an elaborate pattern, Martha would ask the following simple question: If I want a sample of the patterns of each scarf, how large of a sample should I take from each one? The students immediately understand that a very small sample is needed from the single-colored scarf, while in the other case a much larger swath was necessary to actually grasp the fullness of the printed pattern. This activity was adapted for the visually impaired student, where the two tiles in Figure 2 were presented to the visually impaired student, concurrent to the scarf experience. The tile on the left of Figure 2 is very plain and has a smooth surface whereas the one on the right is a very intricate mesh of ropes and ribbons. All students came to the conclusion that a very small sample from the plain scarf or the smooth surface tile was enough to be a representative sample. In the discussion, it was clear to students that a large sample size is not a requirement for a representative sample. 


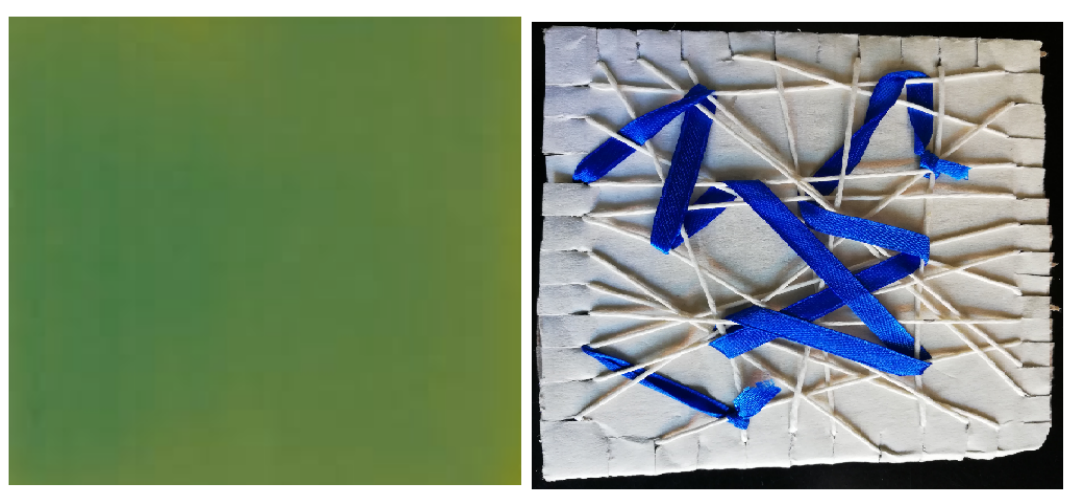

Figure 2. What size of a sample is necessary to get a representative sample?

\section{Hypothesis testing}

The concepts of hypothesis testing, such as one-sided versus a two-sided test, and the meaning of a p-value and level of significance, $\alpha$, presented quite a challenge to all students. Understanding these concepts was the focus of this activity. Seen in Figure 3 is the graphical approach taken for normal visual students on the left and visually impaired students on the right, where for the latter, the labels were translated into Braille with an embossed printing of the graph shown in class.
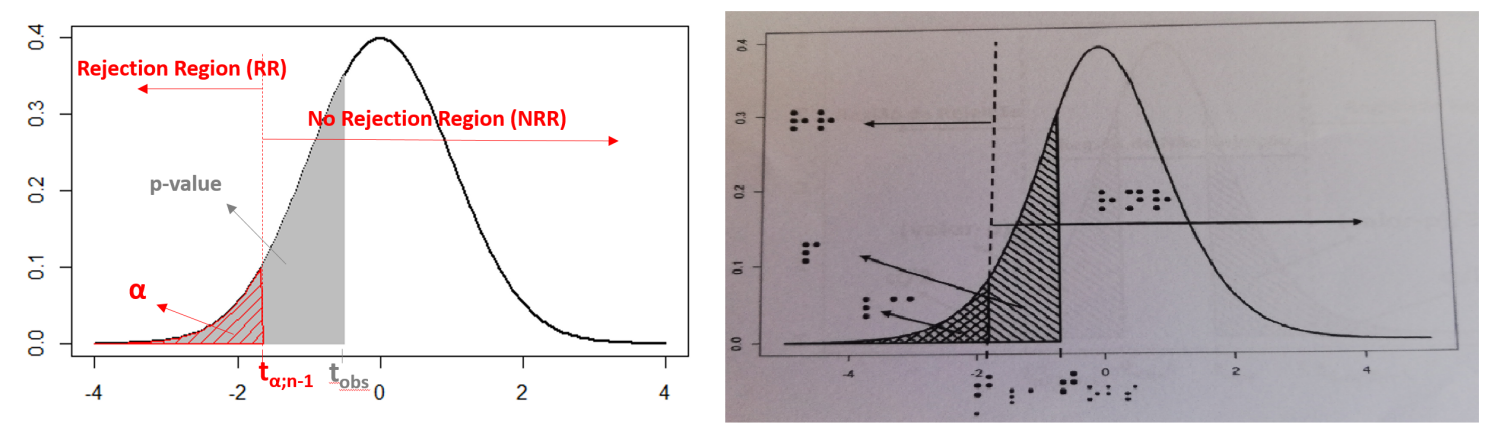

Figure 3. Representation areas in a one-sided test to the left

The final version of the graphs in Figure 3 was constructed sequentially in a PowerPoint presentation for the normal visual students and in a multitude of embossed printings for the visually impaired student. Presenting the two formats in class simultaneously was a challenge and, certainly a process that needs to be perfected in future research. The main conclusions from the discussion in class is that both normal visual and visually impaired students still show some difficulties in grasping these concepts, and further sessions on these subjects were necessary.

The concepts of a distribution and the representation of probabilities as the area under the density curve were concepts that needed some extra time and alternatives approaches with the visually impaired student. For that we used a didactic tactile device called Multiplano, developed for visually impaired learners by Ruben Ferronato (2002), while teaching a Differential and Integral Calculus course at his university. After some adaptations, Figure 4 shows a representation of the density curve drawn using metallic screws, and a p-value for a one-sided test to the right represented by plastic pins. Having different types of materials for the pegs was, in fact, an important detail in distinguishing the density function and the area under the curve. Multiplano was very useful to further explore the concepts in hypotheses testing, but one that is certainly more tailored to one-on-one sessions. 


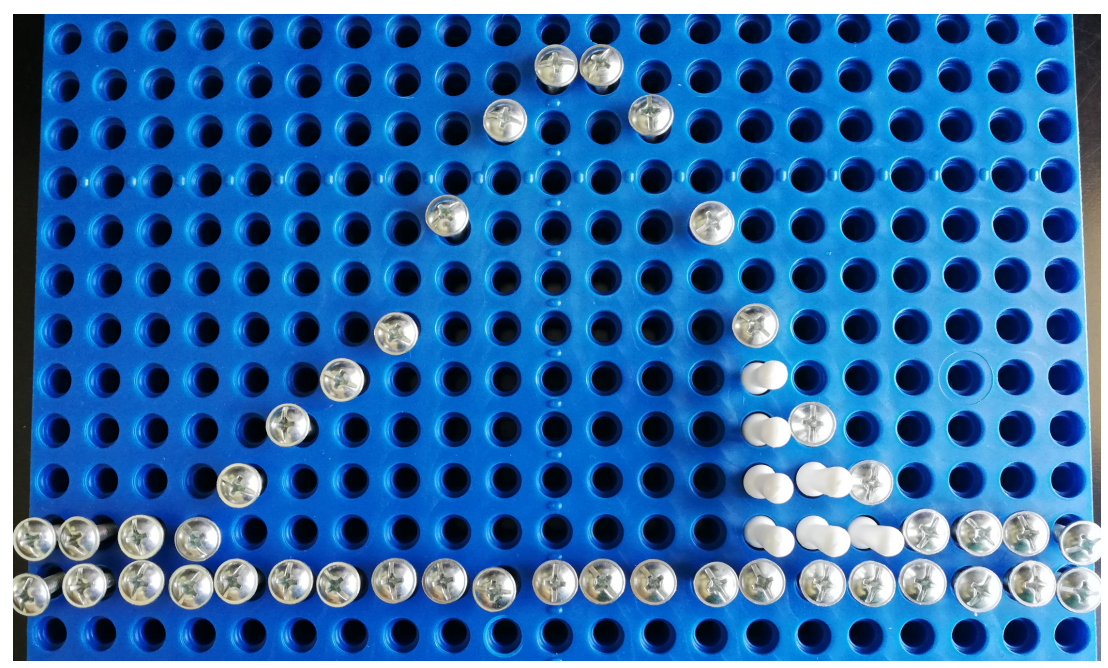

Figure 4. Representation of a p-value in Multiplano

\section{Using a software}

The final challenge for the class was to work with commonly used statistical software in practical classes. Two softwares were crucial in overcoming certain barriers in this learning process, namely the screen reader NVDA (NVDA2019.1.1, 2019) and the statistical software R Studio (R Core Team, 2019). NVDA (Non-Visual Desktop Access) is a free and open source screen reader for the Microsoft Windows operating system. Unfortunately, SPSS, the software selected for class, could not be used because its menus are images and are thus impossible for NVDA to read.

$\mathrm{R}$ Studio also poses some challenges due to the existence of multiple windows when entering the program, which makes NVDA unable to recognize what is written in the different consoles. The way to overcome this difficulty was to teach basic R commands to the visually impaired student and be able to work with some packages that allowed for hearing the results using NVDA. The first R package worth note is "TeachingDemos" where, by using the functions txtStart() and txtStop(), we were able write the commands and outputs produced by $\mathrm{R}$ in a text file, a document that NVDA reads with no difficulty. The second package used was BrailleR, a package that includes the text descriptions of some basic graphs, without the need for emboss printing all the graphics produced. Among many other functions in this package, we emphasize the function $\mathrm{VI}()$ which allows for some of the outputs to be read horizontally. To understand the importance of such a feature, ask someone to read you the Summary, located at the bottom left of Figure 5, the format we usually work in R. Now ask them to read the format of the Summary at the bottom right of Figure 5. It is quite easy to understand that for someone listening to the results, the format on the right is much more straightforward, simpler to follow and preferable.
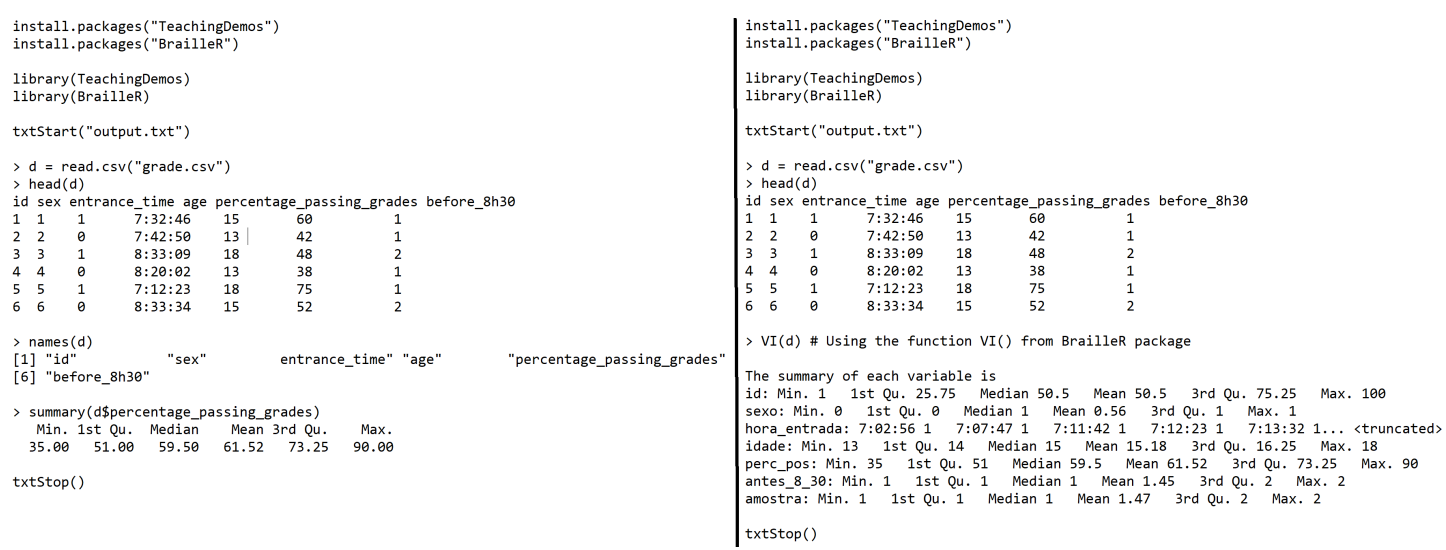

Figure 5. Outputs produced using TeachingDemos and BrailleR packages 
Exploring R with the visually impaired student, while the normal visual students worked in SPSS, allowed the practical classes to take place simultaneously, in which all students, in 4-person groups, had to answer the same challenges.

\section{FINAL COMMENTS}

With an increasingly more diverse corps of students in higher education, teachers and support staff must always stress the importance of inclusion, whether it represents different cultures, languages, or indeed special needs. This case study envisioned Statistical Thinking in a way that visual, aural and tactile senses were employed together.

A very pragmatic non-visual orientation required class planning to emphasize contents received aurally or read in Braille, with activities and challenges designed to engage all students in a variety of formats. Not all the materials used in class using audio, embossed printings, R programming scripts, and Multiplano were successful, but they point in promising directions that can be taken in teaching Statistical Thinking to all types of learners. The common thread for a successful activity was its ability to be performed simultaneously to both the normal visual and visually impaired students. Of important note is that all students were submitted to the same Final Exam, one which the visually impaired student took, obtaining a passing grade for the course.

Future research should address the development of packages in NVDA that would allow the program to automatically read what scripts are produced in RStudio and their outcomes, thus avoiding the need to write them to text files for later reading. For teaching, it would help to publish lecture notes in Statistical Thinking that can simultaneously be used by both the blind and normal visual individuals. This not only would serve to overcome one of the difficulties encountered during classes - which was the ability for both teacher and student be on the same page of the material - but also would nurture the development of Statistical Thinking in students by incorporating this more pragmatic non-visual way of explaining Statistics.

There are still many opportunities to improve the processes founded in this case study, and they are ones worth taking for the good of all students. Somehow, in following the precept that if you do not see the data, try feeling it, either tactilely or aurally, we were made to consider the weight and meaning of every single word employed when teaching Statistical Thinking to students.

\section{REFERENCES}

Aliaga, M. \& Gunderson, B. (2006). Interactive Statistics, 3rd Edition. Upper Saddle River, NJ: Pearson Prentice Hall.

Ferronato, R. (2002). A construção de instrumento de inclusão no ensino da matemática. Master's Thesis in Production Engineering at Universidade Federal de Santa Catarina, Florianópolis, Brazil. URL: https://repositorio.ufsc.br/xmlui/handle/123456789/82939.

NVDA2019.1.1 (2019). Non-Visual Desktop Access a free and open source screen reader developed by NV Access. URL: https://www.nvaccess.org/about-nvda/.

R Core Team (2019). R: A language and environment for statistical computing. R Foundation for Statistical Computing, Vienna, Austria. URL: https://www.R-project.org/. 